\title{
Autoavaliação negativa da saúde em pessoas idosas associada a condições socioeconômicas e de saúde: inquérito populacional em Rio Branco, Acre
}

Negative self-rated health in older people associated with socioeconomic conditions and health: a population survey in Rio Branco, Acre

\author{
Rodolfo Esteban Kelca Usnayo' (ID \\ Gina Torres Rego Monteiro² \\ Cledir de Araújo Amaral ${ }^{3}$ ID \\ Maurício Teixeira Leite de Vasconcellos 4 ID \\ Thatiana Lameira Maciel Amaral' $\mathbb{( D}$
}

\section{Resumo}

Objetivo: Estimar a prevalência de autoavaliação negativa da saúde e sua associação com condições socioeconômicas, sintomas depressivos, funcionalidade e morbidades autorreferidas em pessoas idosas de Rio Branco, Acre. Método: Trata-se de uma pesquisa realizada com dados do Estudo das Doenças Crônicas em Idosos (EDOC-I), um inquérito domiciliar realizado com pessoas a partir de 60 anos residentes em Rio Branco, Acre, Brasil, em 2014 (n=1.016). A autoavaliação negativa da saúde foi definida pelos estratos "ruim" e “muito ruim”. Foram aplicadas as escalas de depressão geriátrica e das atividades da vida diária e instrumentais da vida diária. Como medida de associação da autoavaliação negativa da saúde com variáveis de interesse foi empregada a técnica de regressão logística múltipla. Resultados: A prevalência de autoavaliação negativa da saúde foi de 15,4\%, apresentando associação estatisticamente significativa com sexo feminino (OR:1,72; IC95\%:1,17-2,51), baixa escolaridade (OR:2,33; IC95\%:1,37-3,97), sedentarismo (OR:1,84; IC95\%: 1,08-3,14) e uso de medicamentos (OR:3,01; IC95\%:1,52-5,95). Também se detectou associação com sintomas depressivos (OR:2,55; IC95\%:1,74-3,73), presença de multimorbidades (OR:1,73; IC95\%:1,15-2,61) e dependência total nas atividades instrumentais da vida diária (OR:2,42; IC95\%:1,40-4,17). As morbidades associadas à percepção negativa da saúde foram: asma/ bronquite (OR:2,74; IC95\%:1,61-4,67), insônia (OR:1,80; IC95\%:1,25-2,58) e problemas

\footnotetext{
Universidade Federal do Acre, Centro de Ciências da Saúde e do Desporto, Programa de Mestrado em Saúde Coletiva. Rio Branco, AC, Brasil.

2 Fundação Oswaldo Cruz (Ensp/FIOCRUZ), Escola Nacional de Saúde Pública. Rio de Janeiro, RJ, Brasil.

3 Instituto Federal do Acre, Mestrado Profissional em Educação Profissional e Tecnológica. Rio Branco, AC, Brasil.

4 Fundação Instituto Brasileiro de Geografia e Estatística, Escola Nacional de Ciências Estatísticas, Sociedade para o Desenvolvimento da Pesquisa Científica (Science). Rio de Janeiro, RJ, Brasil.
}

Financiamento da pesquisa: Conselho Nacional de Desenvolvimento Científico e Tecnológico [CNPq Chamada MCTI/CNPQ/MS-SCTIE-DECIT 06/2013, para apoio a pesquisas estratégicas para o Sistema de Saúde pela Rede Brasileira de Avaliação de Tecnologias em Saúde (REBRATS), Processo 401081/2013-3]; Fundação de Amparo à Pesquisa do Acre [FAPAC - Chamada PPSUS 001/2013, do Programa de Pesquisa para o SUS: gestão compartilhada em saúde (MS/CNPq/FAPAC/SESACRE), Processo 6068-14-0000029]. Os autores declaram não haver conflito na concepção deste trabalho.
Palavras-chave: Saúde do Idoso. Autoavaliação. Depressão. Idoso Fragilizado. Inquéritos e Questionários. Medidas de Associação. 
cardíacos (OR:1,77; IC95\%:1,18-2,68). Conclusão: Assim, a autoavaliação negativa da saúde das pessoas idosas sofre influência das condições socioeconômicas e de saúde, sendo um indicador útil para o delineamento de estratégias em saúde que favoreçam o envelhecimento com independência e bem-estar físico e emocional.

\section{Abstract}

Objective: To estimate the prevalence of negative self-rated health and its association with socioeconomic conditions, depressive symptoms, self-reported functionality, and morbidities in older people in Rio Branco, Acre. Method: This is a survey carried out with data from Estudo das Doenças Crônicas em Idosos (EDOC-I - Study of Chronic Diseases in Older People), a household survey carried out with people aged 60 and over living in Rio Branco, Acre, Brazil, in 2014 ( $n=1,016)$. Negative self-rated health was defined by the "bad" and "very bad" strata. The scales of geriatric depression and activities of daily living and instrumental activities of daily living were applied. As a measure of association between negative self-rated health and variables of interest, the multiple logistic regression technique was used. Results: The prevalence of negative self-rated health was $15.4 \%$, with a statistically significant association with females (OR: 1.72; 95\%CI:1.17-2.51), low education (OR:2.33; 95\%CI:1.37-3.97), sedentary lifestyle (OR:1.84; 95\%CI:1.08-3.14), and medication use (OR:3.01; 95\%CI:1.52-5,95). An association was also detected with depressive symptoms (OR:2.55; 95\%CI:1.74-3.73), presence of multimorbidities (OR:1.73; 95\%CI:1.15-2.61), and total dependence on instrumental activities of daily living (OR:2.42; 95\%CI:1.40-4.17). The morbidities associated with the negative perception of health were asthma/bronchitis (OR:2.74; 95\%CI:1.61-4.67), insomnia (OR:1.80; 95\%CI:1.25-2.58), and heart problems (OR:1.77; 95\%CI:1.18-2.68). Conclusion: Therefore, the negative selfrated health of older people is influenced by socioeconomic and health conditions, being a useful indicator for the design of health strategies to favor aging with independence and physical and emotional well-being.

\section{INTRODUÇÃOO}

O envelhecimento populacional é uma realidade também no Brasil, e resulta em alteração no padrão de morbimortalidade. O avançar da idade é inevitável e progressivo, assim como os fenômenos que os acompanham, que são desgaste orgânico, alterações nos aspectos culturais, sociais e emocionais. Essas mudanças influenciam a autoavaliação da saúde, uma vez que, a ideia de envelhecimento, saúde e doença perpassam e sofrem ação do momento sócio-histórico construído por uma variedade de componentes físicos, culturais e afetivos, possibilitando o confundimento de condições clínicas e não-clínicas ${ }^{1}$.

Os fatores considerados no indivíduo, ao classificar seu estado de saúde, ainda não são totalmente definidos, mas incluem diferentes aspectos como indicadores sociodemográficos, condições de saúde e diminuição da funcionalidade entre as pessoas idosas ${ }^{2}$. Deve-se mencionar que a correlação entre a saúde objetiva e a subjetiva tende a diminuir com o avançar da idade. No entanto, diante de condições de incapacidade, presença de morbidades e depressão ocorre o aumento da prevalência de autoavaliação negativa da saúde ${ }^{3}$.

Em uma revisão sistemática, com estudos de 1999 a 2011, a prevalência de autoavaliação negativa da saúde entre pessoas idosas variou de 12,6 a 51,9\%. Na presença de doenças, foi observada associação com uso de medicamentos, renda familiar, internações, consultas médicas, dependência nas atividades da vida diária, presença de sintomas depressivos e ansiosos e insônia ${ }^{4}$.

O entendimento dos fatores associados à autoavaliação negativa da saúde em pessoas idosas possibilita identificar e acompanhar as condições gerais de saúde dessa população. Permite, ainda, comparar seus resultados com os de outras regiões do país, uma vez que, estudos na Amazônia sobre essa temática são escassos. Assim, o objetivo do presente estudo foi estimar a prevalência de 
autoavaliação negativa da saúde e sua associação com condições socioeconômicas, sintomas depressivos, funcionalidade e morbidades autorreferidas em pessoas idosas de Rio Branco, Acre, Brasil.

\section{MÉTODO}

Trata-se de uma pesquisa realizada com dados do Estudo das Doenças Crônicas em Idosos (EDOC-I) ${ }^{5}$, inquérito domiciliar realizado com idosos (a partir de 60 anos) residentes em Rio Branco (AC), Brasil, em 2014. Com plano amostral por conglomerados em dois estágios (setor censitário e domicílio), foram incluídas todas as pessoas idosas residentes nos domicílios sorteados de cada um dos 40 setores censitários investigados. Foram excluídas da pesquisa aquelas com comprometimentos cognitivos, mediante relato dos familiares ou constatação da equipe, que inviabilizassem a comunicação ou o entendimento das perguntas.

O tamanho da amostra do EDOC-I foi determinado pressupondo uma prevalência de $40,0 \%$ de alterações da função renal, com grau de confiança de 95,0\% e erro absoluto de 3,0\%. A amostra final abrangeu 1.016 idosos, que com base nos pesos calibrados das observações permitiram estimativas inferenciais para a população de 23.416 idosos residentes em $1^{\circ}$ de julho de 2014 , em Rio Branco. Maiores informações sobre o plano amostral, instrumentos e protocolos para coletas de dados verificar no artigo metodológico previamente publicado por Amaral et al. ${ }^{5}$.

A coleta de dados ocorreu de abril a setembro de 2014. Foram realizadas entrevistas domiciliares para investigar as condições socioeconômicas, demográficas e de saúde, além de hábitos de vida dos participantes. Também foram realizadas avaliações físicas com medidas antropométricas, de frequência cardíaca e de pressão arterial, além de coleta de amostras de sangue e urina para análises clínicas.

O índice de massa corporal (IMC) foi avaliado segundo os pontos de corte específicos para população idosa: baixo-peso (IMC $<22 \mathrm{~kg} / \mathrm{m}^{2}$ ), eutrófico (IMC entre $22 \mathrm{~kg} / \mathrm{m}^{2}$ e $27 \mathrm{~kg} / \mathrm{m}^{2}$ ) e sobrepeso $\left(\mathrm{IMC}>27 \mathrm{~kg} / \mathrm{m}^{2}\right)$.
A presença de hipertensão arterial foi definida como pressão arterial diastólica (PAD) $\geq 90 \mathrm{mmHg}$ e/ou pressão arterial sistólica (PAS) $\geq 140 \mathrm{mmHg}$ e/ ou uso atual de medicação anti-hipertensiva. Para o diabetes mellitus, foram usados os critérios da American Diabetes Association (ADA): glicose no plasma em jejum $\geq 126 \mathrm{mg} / \mathrm{dl}$, bem como a utilização de hipoglicemiante oral ou de insulina ${ }^{6}$.

A dislipidemia foi definida pela presença de níveis anormais de um ou mais dos seguintes componentes lipídicos do sangue: colesterol total $\geq 200 \mathrm{mg} / \mathrm{dl}$; LDL-C $\geq 160 \mathrm{mg} / \mathrm{dl}$; triglicerídeos $\geq 150 \mathrm{mg} / \mathrm{dl}$; e HDL-C em homens $<40 \mathrm{mg} / \mathrm{dl}$ e mulheres $<50$ $\mathrm{mg} / \mathrm{dl}$, além do relato de uso de medicamentos para redução desses valores ${ }^{7}$.

A multimorbidade foi considerada a partir de dois pontos de corte, um considerou a presença de 2 ou mais doenças crônicas e outro a partir de 3 .

Para a presença de sintomas de depressão foi utilizada a Escala de Depressão Geriátrica Reduzida (EDG). A escala foi adaptada e validade para a população brasileira por Almeida e Almeida ${ }^{8}$. Essa escala apresenta pontuação que varia de 0 a 15 , sendo considerado como caso sugestivo da doença aquele a partir de 6 pontos.

Para avaliar a capacidade funcional foi utilizado Índice de Katz para as Atividades da Vida Diária (AVD) e a escala de Lawton e Brody para as Atividades Instrumentais da Vida Diária (AIVD). A escala de Katz já foi validada para o Brasil ${ }^{9}$ e avalia o nível de autonomia das pessoas idosas no desempenho de seis atividades cotidianas: vestir-se; banhar-se; ir ao banheiro; sentar-se, deitar-se e levantar-se da cama ou cadeira; continência; e alimentar-se. O escore varia entre 0 e 6 pontos, sendo 1 ponto atribuído a cada resposta Sim. Com base na escala de Katz, as pessoas idosas foram classificadas como independentes (6 a 5 pontos), dependentes parciais ( 4 a 3 pontos) ou dependentes totais (menos de 3 pontos).

A escala de Atividades Instrumentais da Vida Diária (AIVD), adaptada para o português brasileiro $^{10}$, é composta por oito atividades: cuidar da casa, lavar a roupa, preparação da comida, ir às compras, utilização do telefone, utilização de transporte, gestão do dinheiro e gestão da medicação. 
Para essa escala, aqueles idosos que atingiram 27 pontos foram classificados como independentes, entre 26-18 pontos com dependência parcial e menos de 18 pontos, como dependentes.

A variável dependente da presente investigação foi obtida pelas respostas à pergunta Em geral, diria que sua saúde é: muito boa, boa, regular, ruim ou muito ruim? Foi definido como autoavaliação negativa da saúde aqueles que indicaram os estratos ruim e muito ruim.

Para o controle da qualidade das informações, foram realizadas entrevistas e avaliações físicas de 30 idosos no estudo piloto ${ }^{5}$.

Os dados foram analisados de forma descritiva e exploratória para avaliar a distribuição e caracterizar a população estudada. As variáveis foram descritas por frequências absolutas e proporções, sendo as diferenças entre proporções estimadas pelo teste qui-quadrado de Pearson.

A análise bivariada foi realizada com objetivo de explorar a associação das diferentes variáveis e o objeto de estudo. Modelos de regressão logística estimaram a magnitude de associação, em odds ratio (OR), entre a variável dependente autoavaliação negativa da saúde e as variáveis independentes.

$\mathrm{Na}$ análise múltipla, foram incluídas as variáveis que apresentaram $p<0,20$ na análise bruta, sendo estimada a magnitude de associação das variáveis ajustadas pelas demais variáveis significativas. Utilizouse o modelo hierárquico, no qual no primeiro nível entraram as variáveis demográficas e socioeconômicas; no segundo, os hábitos de vida e condições de saúde; e no terceiro, as morbidades e fatores relacionados às doenças. As variáveis significativas em uma etapa permaneceram no modelo nas etapas seguintes. Adicionalmente, realizou-se análise de regressão múltipla para a presença de multimorbidade, sintomas depressivos e capacidade funcional. O nível de significância considerado foi de $\alpha=0,05$.

Todas as análises levaram em conta o efeito do desenho amostral e os pesos calibrados das observações, a partir dos quais foram definidas as proporções. As frequências das observações na amostra foram expressas por 'n' e as inferências populacionais, por ' $\mathrm{N}$ '.
O EDOC atendeu aos dispositivos da Resolução CNS 466/2012 que trata da ética em pesquisas envolvendo seres humanos e foi aprovado pelo Comitê de Ética em Pesquisas (CEP) da Universidade Federal do Acre sob o parecer n. 1.610.359.

\section{RESULTADOS}

A prevalência de autoavaliação negativa entre pessoas idosas de Rio Branco, em 2014, foi de 15,4\%. A prevalência no estrato regular foi de $47,9 \%$ e a de autoavaliação positiva foi de 36,7\%. Na análise da autoavaliação negativa, maiores prevalências foram observadas nos indivíduos do sexo feminino, escolaridade até o ensino fundamental e sedentários $(p<0,05)$ (Tabela 1).

Maiores prevalências de percepção negativa da saúde também foram observadas entre as pessoas idosas que utilizam medicamentos, com histórico de internação nos 12 meses anteriores à entrevista e entre aqueles que referiram sofrer de asma/bronquite, insônia, má circulação, problemas cardíacos e osteoporose (todos $\operatorname{com} p<0,05)$ (Tabela 2).

Na presença de sintomas depressivos e dependência nas AIVD, a prevalência de autoavaliação negativa da saúde foi superior a 25,0\%. A autoavaliação negativa também obteve maior prevalência entre as pessoas idosas com multimorbidade avaliada para dois ou mais morbidades crônicas e para três ou mais $(p<0,05)$ (Tabela 2).

A análise multivariada ajustada por nível hierárquico, no nível distal, revelou associações estatisticamente significativas da autoavaliação negativa da saúde com sexo e escolaridade. No nível intermediário, detectou-se associação com sedentarismo e, no nível proximal, com o uso de medicação, presença de asma/bronquite, insônia e problemas cardíacos (ICC, arritmias, infarto agudo do miocárdio) (Tabela 3).

A presença de sintomas depressivos, multimorbidades e dependência funcional resultaram em aumento na chance de o indivíduo autoavaliar negativamente sua saúde, após ajuste por variáveis potencialmente confundidoras (Tabela 4). 
Tabela 1. Prevalência de autoavaliação negativa da saúde segundo as características sociodemográficas e hábitos de vida em pessoas idosas. Rio Branco, Acre, Brasil, 2014.

\begin{tabular}{|c|c|c|c|c|}
\hline \multirow{2}{*}{ Variáveis } & \multirow{2}{*}{$\frac{\text { Total }}{\mathrm{N}}$} & \multicolumn{2}{|c|}{ Autoavaliação Negativa da Saúde } & \multirow{2}{*}{$p$-valor } \\
\hline & & $\mathrm{n}$ & $\mathrm{N}(\%)$ & \\
\hline Total & 23.416 & 1.016 & $3.610(15,4)$ & \\
\hline Idade (anos) & & & & 0,081 \\
\hline $60-79$ & 20.081 & 842 & $2.905(14,5)$ & \\
\hline 80 e mais & 3.335 & 174 & $705(21,2)$ & \\
\hline Sexo & & & & 0,009 \\
\hline Masculino & 10.896 & 418 & $1.283(11,8)$ & \\
\hline Feminino & 12.520 & 598 & $2.327(18,6)$ & \\
\hline Situação Conjugal* & & & & 0,453 \\
\hline Vive em união & 9.086 & 376 & $1.271(14,0)$ & \\
\hline Não vive em união & 14.172 & 633 & $2.277(16,1)$ & \\
\hline Cor da pele & & & & 0,591 \\
\hline Branca & 5.614 & 250 & $931(16,6)$ & \\
\hline Não Branca & 17.802 & 766 & $2.679(15,0)$ & \\
\hline Escolaridade* & & & & 0,001 \\
\hline Ensino médio e superior & 3.789 & 156 & $299(7,9)$ & \\
\hline Até o ensino fundamental & 19.434 & 852 & $3.287(16,9)$ & \\
\hline Atividade física* & & & & 0,003 \\
\hline Sim & 3.577 & 151 & $293(8,2)$ & \\
\hline Não & 19.839 & 865 & $3.317(16,7)$ & \\
\hline Tabagismo* & & & & 0,882 \\
\hline Não fumante & 6.763 & 294 & $1.020(15,1)$ & \\
\hline Fumante/ Ex-fumante & 16.653 & 722 & $2.590(15,6)$ & \\
\hline
\end{tabular}

* As diferenças em relação ao total são decorrentes de falta de informação na variável; ${ }^{a}$ qui-quadrado de Pearson; $\mathrm{N}=$ inferência populacional com base no delineamento amostral.

Tabela 2. Prevalência de autoavaliação negativa da saúde segundo condições de saúde, presença de morbidades, multimorbidade, sintomas depressivos e funcionalidade em pessoas idosas. Rio Branco, Acre, Brasil, 2014.

\begin{tabular}{|c|c|c|c|c|}
\hline \multirow{2}{*}{ Variáveis } & \multirow{2}{*}{$\frac{\text { Total }}{\mathrm{N}}$} & \multicolumn{2}{|c|}{ Autoavaliação Negativa da Saúde } & \multirow{2}{*}{$p$-valor $r^{\mathrm{a}}$} \\
\hline & & $\mathrm{n}$ & $\mathrm{N}(\%)$ & \\
\hline Uso de medicação & & & & $<0,001$ \\
\hline Não & 5.871 & 237 & $332(5,7)$ & \\
\hline $\operatorname{Sim}$ & 17.545 & 779 & $3.278(18,7)$ & \\
\hline $\operatorname{IMC}\left(\mathrm{kg} / \mathrm{m}^{2}\right)^{*}$ & & & & 0,100 \\
\hline$<22$ & 2.789 & 128 & $583(20,9)$ & \\
\hline 22 a 27 & 10.042 & 432 & $1.292(12,9)$ & \\
\hline$>27$ & 9.204 & 397 & $1.568(17,0)$ & \\
\hline Obesidade central (CC)* & & & & 0,410 \\
\hline $\mathrm{CC} \leq 102 \mathrm{M}$ ou $\leq 88 \mathrm{~F}$ & 14.381 & 610 & $2.120(14,7)$ & \\
\hline $\mathrm{CC}>102 \mathrm{M}$ ou $>88 \mathrm{~F}$ & 7.871 & 357 & $1.364(17,3)$ & \\
\hline Internação nos últimos 12 meses* & & & & 0,003 \\
\hline Não & 18.868 & 818 & $2.614(13,9)$ & \\
\hline $\operatorname{Sim}$ & 3.746 & 162 & $823(22,0)$ & \\
\hline
\end{tabular}


Continuação da Tabela 2

\begin{tabular}{|c|c|c|c|c|}
\hline \multirow{2}{*}{ Variáveis } & \multirow{2}{*}{$\begin{array}{l}\text { Total } \\
\mathrm{N}\end{array}$} & \multicolumn{2}{|c|}{ Autoavaliação Negativa da Saúde } & \multirow{2}{*}{$p$-valor ${ }^{2}$} \\
\hline & & $\mathrm{n}$ & $\mathrm{N}(\%)$ & \\
\hline Hipertensão arterial* & & & & 0,251 \\
\hline Não & 5.458 & 235 & $1.002(18,4)$ & \\
\hline Sim & 17.385 & 759 & $2.584(14,9)$ & \\
\hline Diabetes* & & & & 0,226 \\
\hline Não & 18.843 & 820 & $2.740(14,5)$ & \\
\hline $\operatorname{Sim}$ & 3.834 & 166 & $705(18,4)$ & \\
\hline Dislipidemia* & & & & 0,930 \\
\hline Não & 4.585 & 198 & $696(15,2)$ & \\
\hline Sim & 18.031 & 785 & $2.782(15,4)$ & \\
\hline \multicolumn{5}{|l|}{ Morbidades autorreferidas } \\
\hline Asma/Bronquite & & & & $<0,001$ \\
\hline Não & 21.067 & 913 & $2.853(13,5)$ & \\
\hline Sim & 2.275 & 100 & $725(31,9)$ & \\
\hline Insônia* & & & & 0,001 \\
\hline Não & 15.333 & 658 & $1.904(12,4)$ & \\
\hline Sim & 8.039 & 356 & $1.706(21,2)$ & \\
\hline Má circulação & & & & 0,047 \\
\hline Não & 2.018 & 632 & $2.018(13,6)$ & \\
\hline Sim & 8.437 & 379 & $1.518(18,0)$ & \\
\hline Problemas cardíacos* & & & & $<0,001$ \\
\hline Não & 19.843 & 858 & $2.547(12,8)$ & \\
\hline Sim & 3.245 & 144 & $880(27,1)$ & \\
\hline Acidente Vascular Encefálico* & & & & 0,513 \\
\hline Não & 22.313 & 967 & $3.480(15,6)$ & \\
\hline $\operatorname{Sim}$ & 1.026 & 46 & $130(12,7)$ & \\
\hline Osteoporose* & & & & 0,007 \\
\hline Não & 19.473 & 829 & $2.703(13,9)$ & \\
\hline $\operatorname{Sim}$ & 3.453 & 164 & $775(22,4)$ & \\
\hline Depressão (EDG)* & & & & $<0,001$ \\
\hline Não & 15.666 & 671 & $1.658(10,6)$ & \\
\hline $\operatorname{Sim}$ & 7.558 & 337 & $1.908(25,2)$ & \\
\hline \multicolumn{5}{|l|}{ Multimorbidade* } \\
\hline Não & 10.019 & 419 & $1.112(11,1)$ & 0,002 \\
\hline $\operatorname{Sim}(\geq 2)$ & 13.398 & 597 & $2.498(18,6)$ & \\
\hline Não & 14.786 & 627 & $1.708(11,6)$ & $<0,001$ \\
\hline $\operatorname{Sim}(\geq 3)$ & 8.630 & 389 & $1.902(22,0)$ & \\
\hline AVD* & & & & 0,898 \\
\hline Independente & 17.539 & 760 & $2.684(15,3)$ & \\
\hline Dependência parcial & 4.000 & 176 & $584(14,6)$ & \\
\hline Dependência total & 1.771 & 75 & $299(16,9)$ & \\
\hline AIVD & & & & $<0,001$ \\
\hline Independente & 11.908 & 490 & $1.309(11,0)$ & \\
\hline Dependência parcial & 9.079 & 412 & $1.657(18,3)$ & \\
\hline
\end{tabular}

* As diferenças em relação ao total são decorrentes de falta de informação na variável; a qui-quadrado de Pearson; $\mathrm{N}$ = inferência populacional com base no delineamento amostral. IMC = Índice de Massa Corporal; EDG = escala de depressão geriátrica; AIVD = atividades instrumentais da vida diária; $\mathrm{AVD}=$ atividades da vida diária. 
Tabela 3. Modelo hierárquico da autoavaliação negativa da saúde segundo as variáveis independentes em pessoas idosas. Rio Branco, Acre, Brasil, 2014.

\begin{tabular}{|c|c|c|}
\hline Variáveis & $\mathrm{OR}_{\text {Bruta }}(\mathrm{IC} 95 \%)$ & $\mathrm{OR}_{\text {Aiust }}(\mathrm{IC} 95 \%)$ \\
\hline \multicolumn{3}{|l|}{ Nível distal } \\
\hline Idade ( $\geq 80$ vs. $60-79)$ & $1,59(0,94-2,68)$ & $1,47(0,86-2,51)$ \\
\hline Sexo (feminino vs. masculino) & $1,71(1,15-2,55)$ & $1,72(1,17-2,51)$ \\
\hline Escolaridade (até ens. fundamental vs. $\geq$ ens. médio) & $2,37(1,40-4,01)$ & $2,33(1,37-3,97)$ \\
\hline \multicolumn{3}{|l|}{ Nível Intermediário } \\
\hline Atividade física (sedentário vs. ativo) & $2,25(1,33-3,78)$ & $1,84(1,08-3,14)$ \\
\hline \multicolumn{3}{|l|}{$\operatorname{IMC}\left(\mathrm{kg} / \mathrm{m}^{2}\right)$} \\
\hline$<22$ vs. $22-27$ & $1,79(1,15-2,79)$ & $1,58(0,99-2,52)$ \\
\hline$>27$ vs. $22-27$ & $1,39(0,87-2,22)$ & $1,31(0,82-2,10)$ \\
\hline \multicolumn{3}{|l|}{ Nível Proximal } \\
\hline Uso de medicação (sim vs. não) & $3,84(2,11-6,98)$ & $3,01(1,52-5,95)$ \\
\hline Internação (sim vs. não) & $1,75(1,22-2,51)$ & $1,31(0,83-2,09)$ \\
\hline Asma/Bronquite (sim vs. não) & $2,98(1,82-4,89)$ & $2,74(1,61-4,67)$ \\
\hline Insônia (sim vs. não) & $1,90(1,30-2,78)$ & $1,80(1,25-2,58)$ \\
\hline Má circulação (sim vs. não) & $1,39(1,00-1,94)$ & $1,05(0,76-1,45)$ \\
\hline Problemas cardíacos (sim vs. não) & $2,53(1,73-3,70)$ & $1,77(1,18-2,68)$ \\
\hline Osteoporose (sim vs. não) & $1,79(1,18-2,74)$ & $1,19(0,73-1,96)$ \\
\hline
\end{tabular}

$\mathrm{OR}=$ Odds Ratio; IC = intervalo de confiança. $\mathrm{OR}$ = Odds Ratio ajustada. Nível distal ajustado pelas variáveis distais entre si; Nível intermediário ajustado pelas variáveis intermediárias entre si e pelas variáveis significativas do nível distal; Nível proximal, ajustado pelas variáveis proximais e pelas variáveis significativas dos níveis distal e intermediário.

Até ens. fundamental $=$ escolaridade até ensino fundamental; $\geq$ ens. médio $=$ escolaridade maior ou igual ao ensino médio; IMC = Índice de Massa Corporal.

Tabela 4. Análise de regressão logística da presença de multimorbidade, sintomas depressivos e funcionalidade com autoavaliação negativa da saúde em pessoas idosas. Rio Branco/Acre, Brasil, 2014.

\begin{tabular}{|c|c|c|c|}
\hline Variáveis & $\begin{array}{l}\mathrm{OR}_{\text {Bruto }}(\mathrm{IC} 95 \%) \\
\text { (modelo } 1) *\end{array}$ & $\begin{array}{l}\mathrm{OR}_{\text {Ajust }}(\mathrm{IC} 95 \%) \\
\text { (modelo 2)** }\end{array}$ & $\begin{array}{l}\mathrm{OR}_{\text {Ajust }}(\mathrm{IC} 95 \%) \\
\text { (modelo 3)*** }\end{array}$ \\
\hline \multicolumn{4}{|l|}{ Depressão (EDG) } \\
\hline Sim vs. Não & $2,85(1,91-4,26)$ & $2,69(1,85-3,93)$ & $2,55(1,74-3,73)$ \\
\hline \multicolumn{4}{|l|}{ Atividades instrumentais da vida diária } \\
\hline Dependente parcial vs. Independente & $1,81(1,23-2,65)$ & $1,71(1,12-2,60)$ & $1,63(1,06-2,51)$ \\
\hline Dependente total vs. Independente & $2,82(1,71-4,65)$ & $2,64(1,51-4,62)$ & $2,42(1,40-4,17)$ \\
\hline \multicolumn{4}{|l|}{ Multimorbidade $(\geq 2)$} \\
\hline Sim vs. Não & $1,84(1,26-2,68)$ & $1,69(1,14-2,51)$ & $1,73(1,15-2,61)$ \\
\hline \multicolumn{4}{|l|}{ Multimorbidade $(\geq 3)$} \\
\hline Sim vs. Não & $2,16(1,63-2,87)$ & $1,97(1,44-2,72)$ & $1,93(1,37-2,72)$ \\
\hline
\end{tabular}

*modelo 1: bruto; ${ }^{* *}$ modelo 2: ajustado por sexo e idade. ${ }^{* * *}$ modelo 3: ajustado por sexo, idade, escolaridade e prática de atividade física; EDG = escala de depressão geriátrica. 


\section{DISCUSSÃO}

Foi identificada uma prevalência moderada de autoavaliação negativa da saúde $(15,4 \%)$ entre as pessoas idosas de Rio Branco, Acre, em 2014, as quais consideraram sua saúde ruim ou muito ruim. A percepção negativa da própria saúde foi associada aos sintomas depressivos e à dependência nas atividades instrumentais da vida diária. Adicionalmente, foram observadas associações com sexo feminino, baixa escolaridade, sedentarismo e uso de medicamentos, além de associação com autorrelato de asma/bronquite, insônia, problemas cardíacos e multimorbidades.

Em comparação com resultados de alguns estudos internacionais ${ }^{11,12}$, a prevalência de autoavaliação negativa da saúde em pessoas idosas de Rio Branco é muito maior que o observado na Argentina e inferior ao apresentado no México. Em Córdoba, Argentina, no ano de 2011, estudo com 436 idosos revelou uma prevalência de $8,3 \%{ }^{11}$. No entanto, no México, em 2012, um estudo com 8.874 idosos de 60 anos e mais detectou uma prevalência de $18,1 \%{ }^{12}$.

As pessoas idosas de Rio Branco apresentam maiores prevalências de percepção negativa da saúde na comparação com resultados de estudos nacionais ${ }^{13-15}$. Dados de 1.344 pessoas, com 60 anos ou mais da cidade de São Paulo, em 2010, a prevalência de autoavaliação negativa da saúde foi de $7,8 \%{ }^{13}$. Na cidade de Campinas, São Paulo, a prevalência entre indivíduos da mesma faixa etária, entre 2008 e 2009, foi de 10,9\% $0^{14}$. Em Minas Gerais, com 686 idosos, a prevalência foi de $13,5 \%{ }^{15}$.

A comparação de estudos sobre autoavaliação de saúde deve ser feita com cautela, visto que muitos estudos incluem a categoria regular como avaliação negativa, superestimando os valores de prevalência e, consequentemente, comprometendo as estimativas de associações. Recomenda-se utilizar exclusivamente as categorias ruim e muito ruim na definição de percepção negativa da saúde ${ }^{4}$.

Dentre as condições associadas à autoavaliação negativa da saúde, as mulheres idosas apresentaram pior avaliação da própria saúde. Elas aparecem como maioria, fenômeno de feminização do envelhecimento, decorrente da expectativa de vida superior à dos homens, e também representam a maior frequência de autoavaliação negativa da saúde em relação aos homens ${ }^{16}$.

Em estudo realizado em São Paulo ${ }^{13}$, a chance de autoavaliação negativa da saúde foi quase duas vezes superior no sexo feminino em relação ao masculino, corroborando os achados do presente estudo. O que implica na existência de um paradoxo a ser enfrentado pelas mulheres ao viver mais, porém em piores condições de saúde, que em parte pode ser explicada por menores níveis de escolaridade e de tempo para atividades de lazer, além das razões de ordem biológica, a exemplo da função reprodutiva, uma vez que as mulheres vivenciam uma fase pós-menopáusica delicada, embora com baixo risco de morte ${ }^{17}$.

As desigualdades não se restringem à variável sexo, dados da Pesquisa de Orçamentos Familiares (POF 2008 - 2009) revelam que o acesso aos serviços básicos é pior entre aqueles de baixa renda e menor escolaridade ${ }^{18}$, sendo no presente estudo observada associação de menor escolaridade com a autoavaliação negativa da saúde entre as pessoas idosas. Em nível global, as desigualdades sociais em saúde são problemas que afetam todos, visto que, independentemente do país em que residem, os indivíduos que convivem com piores condições socioeconômicas são os mais susceptíveis à ocorrência de diversos problemas de saúde e, portanto, pior avaliação dela ${ }^{19}$.

Um estudo nacional ${ }^{20}$, que analisou dados de 1998 a 2013, apontou uma chance 7 a 9 vezes maior de autoavaliação negativa da saúde nos brasileiros com baixo nível educacional quando comparado aos com nível superior de escolaridade. Vale destacar que apesar do progresso dos níveis educacionais nesse período, não houve redução na prevalência da percepção negativa da própria saúde, apontando a necessidade de melhoria das condições crônicas e da saúde mental das pessoas de modo a repercutir positivamente na autopercepção da saúde do brasileiro.

Em relação à associação observada entre uso de medicamentos e percepção negativa da saúde, duas perspectivas devem ser consideradas: a primeira é que uso de medicamentos indica a presença de 
morbidades, o que explica pior autoavaliação de saúde; outra importante consideração é que os efeitos adversos decorrentes de medicação podem repercutir em pior percepção da mesma ${ }^{21}$.

Estudo realizado em Florianópolis, em 2009, com 1.705 idosos revelou a prevalência de polifarmácia de $32,0 \%$, sendo os grupos de medicamentos mais utilizados aqueles indicados para o sistema cardiovascular, trato alimentar e metabolismo e sistema nervoso ${ }^{22}$. Destaca-se a necessidade de maiores cuidados sobre a utilização de medicamentos em pessoas idosas de modo a assegurar a adesão e evitar complicações.

A prevalência de doenças cardiovasculares (DCV) tem aumentado na população idosa em parte devido ao aumento da expectativa de vida e à maior exposição aos fatores de risco ${ }^{23}$. Nos Estados Unidos, cerca de $82,0 \%$ de todas as mortes em pessoas idosas $(\geq 65$ anos) são atribuídas às DCV, e essas doenças possuem importante peso na ocorrência de incapacidades, declínio funcional, custos em saúde e declínio da percepção da saúde, confirmando os achados da presente investigação, o que impõe a necessidade de garantia de uma expectativa de vida ativa e não apenas de sobrevivência das pessoas idosas ${ }^{24}$. No Brasil, a DCV é a principal causa de morte, no entanto, tem demostrado tendência de redução, principalmente, na faixa etária de 50 a 69 anos $^{25}$.

$\mathrm{Na}$ presente pesquisa, a asma/bronquite apresentou-se associada à autoavaliação negativa da saúde, a exemplo do observado em estudo realizado em Montes Claros, Minas Gerais, em que o autorrelato de asma foi três vezes maior nos indivíduos com autoavaliação negativa da saúde ${ }^{15}$.

Se a presença de uma única morbidade pode resultar em alteração na percepção da própria saúde, a ocorrência de multimorbidade é ainda mais impactante, visto que o maior número de doenças gera complicações físicas, sociais e mentais nas pessoas idosas, resultando em piora da autoavaliação da saúde ${ }^{26}$. Dados de um estudo de revisão identificaram que a prevalência de multimorbidade em pessoas idosas no Brasil varia de $30,7 \%$ a $57,0 \%$, estando associada às condições socioeconômicas, demográficas, estilo de vida e estrutura familiar ${ }^{27}$. Em outro estudo com pessoas idosas, dos municípios ao norte do Rio Grande do Sul, a presença de multimorbidade aumentou em $29,0 \%$ a autopercepção negativa da saúde ${ }^{28}$.

A associação entre sintomas de depressão e autoavaliação negativa da saúde identificada entre pessoas idosas no presente estudo também foi observada em outro trabalho ${ }^{14}$. Além das próprias condições de saúde física, as condições psíquicas têm importante efeito na percepção da saúde. Dados da Organização Mundial de Saúde revelam que a população idosa alcançará $22,0 \%$ da população mundial em 2050, sendo importante a saúde mental e o bem-estar nesse momento da vida, visto que 15,0\% dos idosos ( $\geq 60$ anos) sofrem de algum distúrbio mental, e destes, $7,0 \%$ em decorrência de depressão ${ }^{29}$. A depressão é um transtorno comum em idosos e afeta sua capacidade funcional, associando-se às doenças somáticas que conduzem ao isolamento social e repercutem em pior qualidade de vida.

Em Montes Claros, Minas Gerais, a presença de depressão esteve associada ao aumento em duas vezes da autoavaliação negativa da saúde ${ }^{30}$. Dados do inquérito EpiFloripa Idoso, com 1.656 idosos, associou a autoavaliação ruim da saúde com sintomas de depressão (RP=2,64; IC95\% 1,82-3,83) e de dependência funcional ( $\mathrm{RP}=1,83$; IC95\% 1,43-2,33) ${ }^{31}$. À medida que a população envelhece, aumenta a prevalência das doenças crônicas e incapacitantes, levando a alterações nas habilidades da pessoa que podem provocar mudanças consideráveis no desempenho das atividades diárias ${ }^{32}$.

O envelhecimento pode resultar em perda da independência devido a restrições de mobilidade, fragilidade e diminuição da aptidão funcional e habilidades cognitivas, tendo importância programas e políticas próprias para manutenção dos idosos saudáveis e independentes por toda a vida ${ }^{33}$. Segundo Campos et al. ${ }^{34}$, a prevalência de incapacidade funcional no Brasil varia de $13,2 \%$ a $85,0 \%$.

Em meio à transição demográfica, pesquisadores e profissionais de saúde devem se concentrar nas interações entre capacidade funcional, envelhecimento e morbidade e sua relevância para as metas centradas no paciente. Vale destacar que os processos que contribuem para a suscetibilidade à doença e ao declínio no status funcional são inerentes 
à idade, sendo que dentre os componentes sistêmicos e celulares mais importantes para a capacidade funcional estão a disfunção mitocondrial, estresse oxidativo, manuseio anormal de cálcio, inflamação crônica, senescência celular, produção de matriz extracelular, perda de estruturas teloméricas e pouca capacidade de reparo do DNA ${ }^{35}$.

Vale considerar que o presente estudo apresenta limitações pontuais e, também, pontos fortes que devem ser considerados. Uma primeira limitação é o viés de sobrevivência, inerente aos estudos transversais com pessoas idosas; contudo, ressalta-se o caráter exploratório das análises ora apresentadas e a não pretensão de realizar inferências causais. Por outro lado, mostra-se como ponto forte do estudo a amostragem probabilística cujas estimativas são inferenciais para a população idosa do município de Rio Branco. Além disso, a exclusão de indivíduos com capacidade cognitiva comprometida poderia levar a erros nas estimativas de funcionalidade, uma vez que esses têm maior probabilidade de dependência funcional.

Outro ponto, que se deve reconhecer como limitador dos achados é o uso de medidas autorreferidas para algumas condições de saúde que podem subestimar ou superestimar a prevalência. Ademais, a definição de algumas morbidades se deu por meio de critérios clínicos e laboratoriais, portanto, trata-se de um estudo com dados objetivos e subjetivos das condições de saúde para avaliação da autoavaliação negativa de saúde, depressão e incapacidade funcional em pessoas idosas. Essa

\section{REFERÊNCIAS}

1. Fonseca MGUP, Firmo JOA, Loyola Filho AI, Uchôa E. Papel da autonomia na auto-avaliação da saúde do idoso. Rev Saúde Pública. 2010;44(1):159-65.

2. Santos EC, Couto BM, Bastone AC. Factors associated with negative self-assessment of health in elderly enrolled in Primary Care Units. ABCS Health Sc.i 2018;43(1):47-54.

3. Machón M, Vergara I, Dorronsoro M, Vrotsou K, Larrañaga I. Self-perceived health in functionally independent older people: associated factors. BMC Geriatr. 2016;16:1-9. tríade perigosa repercute na qualidade de vida e no bem-estar deste grupo.

\section{CONCLUSÃO}

A população idosa de Rio Branco apresenta moderada prevalência de avaliação negativa da própria saúde e essa autoavaliação está associada à depressão e à dependência nas atividades instrumentais da vida diária, assim como ao autorrelato de asma/bronquite, insônia, problemas cardíacos e multimorbidades. Também foi observada associação com o sexo feminino, baixa escolaridade, sedentarismo e uso de medicamentos.

Ações de monitoramento das condições físicas e psicológicas devem ser uma constante na atenção à saúde das pessoas idosas, sendo que a avaliação da capacidade funcional, dos sintomas de depressão e da autoavaliação de saúde precisam ser medidos e utilizados para nortear ações preventivas. A autoavaliação da saúde é um indicador importante do status de saúde físico e mental e pode ser usado em estudos e no acompanhamento da saúde da população idosa.

A utilização do presente achado pode ser útil para o delineamento de estratégias em saúde que favoreçam o envelhecimento com independência e bem-estar físico e emocional. Outras pesquisas devem ser realizadas com métodos que permitam a verificação de causalidade.

Editado por: Maria Luiza Diniz de Sousa Lopes
4. Pagotto V, Bachión MM, Silveira EA. Autoavaliação da saúde por idosos brasileiros: revisão sistemática da literatura. Rev Panam Salud Publica. 2013;33(4):302-10.

5. Amaral TLM, Amaral CA, Portela MC, Monteiro GTR, Vasconcellos MTL. Estudo das Doenças Crônicas (Edoc): aspectos metodológicos. Rev Saúde Pública. 2019;53:1-8. Disponível em: https://doi. org/10.11606/s1518-8787.2019053000847 .

6. Sociedade Brasileira de Diabetes. Diretrizes da Sociedade Brasileira de Diabetes: 2013-2014. Organizadores: José Egidio Paulo de Oliveira, Sergio Vencio. São Paulo: AC Farmacêutica; 2014. 
7. Xavier HT, Izar MC, Faria Neto RJ, Assad MH, Rocha VZ, Sposito AC, et al. V Diretriz Brasileira de Dislipidemias e Prevenção da Aterosclerose. Arq Bras Cardiol. 2013;101(supl 1):1-20. Disponível em: https:// doi.org/10.5935/abc.2013S010.

8. Almeida OP, Almeida SA. Short versions of the geriatric depression scale: a study of their validity for the diagnosis of a major depressive episode according to ICD-10 and DSM-IV. Int J Geriatr Psychiatry. 1999;14(10):858-65.

9. Lino VTS, Pereira SEM, Camacho LAB, Ribeiro Filho ST, Buksman S. Adaptação transcultural da Escala de Independência em Atividades da Vida Diária (Escala de Katz). Cad Saúde Pública. 2008;24(1):103-12.

10. Santos RL, Virtuoso Júnior JS. Confiabilidade da versão brasileira da escala de Atividades Instrumentais da Vida Diária. Rev Bras Promoç Saúde. 2008;21(4):290-6.

11. Peláez E, Débora AL, Delia CE. Factores asociados a la autopercepción de salud en adultos mayores. Rev Cubana Salud Pública. 2015;41(4):638-48. Disponível em: http://scielo.sld.cu/scielo.php?script=sci_ arttext\&pid=S0864-34662015000400007\&lng=es\&t $\operatorname{lng}=\mathrm{es}$.

12. Vázquez EB, Niño JAF, Garcia CIA. Autopercepción de la salud, presencia de comorbilidades y depresión en adultos mayores mexicanos: propuesta y validación de un marco conceptual simple. Biomédica. 2017;37(supl 1):92-103.

13. Antunes JLF, Chiavegatto Filho ADPC, Duarte YAO, Lebrão ML. Desigualdades sociais na autoavaliação de saúde dos idosos da cidade de São Paulo. Rev Bras Epidemio.1 2018;21(supl 2):e180010.

14. Borim FSA, Neri AL, Francisco PMSB, Barros MBA. Dimensões da autoavaliação de saúde em idosos. Rev Saúde Pública. 2014;48(5):714-22.

15. Medeiros SM, Silva LSR, Carneiro JA, Ramos GCF, Barbosa ATF, Caldeira AP. Fatores associados à autopercepção negativa da saúde entre idosos não institucionalizados de Montes Claros, Brasil. Ciênc Saúde Colet. 2016;21(11):3377-86.

16. Confortin SC, Giehl MWC, Antes DL, Schneider IJC, Orsi E. Autopercepção positiva de saúde em idosos: estudo populacional no Sul do Brasil. Cad Saúde Pública. 2015;31(5):1049-60.

17. Assari S. Gender differences in the predictive role of self-rated health on short-term risk of mortality among older adults. SAGE Open Med. 2016;4:1-8.

18. Melo NCV, Ferreira MAM, Teixeira KMD. Condições de vida dos idosos no brasil: uma análise a partir da renda e nível de escolaridade. Oikos Fam Soc Debate. 2014;25(1):4-19.
19. Barreto ML. Desigualdades em Saúde: uma perspectiva global. Ciênc Saúde Colet. 2017;22(7):2097-108.

20. Andrade FCD, Mehta JD. Increasing educational inequalities in self-rated health in Brazil, 1998-2013. PLoS ONE. 2018;13(4):e0196494.

21. Lenander C, Bondesson A, Viberg N, Beckman A, Midlöv P. Effects of medication reviews on use of potentially inappropriate medications in elderly patients; a cross-sectional study in Swedish primary care. BMC Health Serv Res. 2018;18(1):1-9.

22. Pereira KG, Peres MA, Iop D, Boing AC, Boing AF, Aziz M, et al. Polifarmácia em idosos: um estudo de base populacional. Rev Bras Epidemiol. 2017;20(2):335-44.

23. Massa KHC, Duarte YAO, Chiavegatto Filho ADP. Análise da prevalência de doenças cardiovasculares e fatores associados em idosos, 2000-2010. Ciênc Saúde Colet. 2019;24(1):105-14.

24. Yazdanyar A, Newman AB. The burden of cardiovascular disease in the elderly: morbidity, mortality, and costs. Clin Geriatr Med. 2009;25(4):563-77.

25. Oliveira GMM, Brant LCC, Polanczyk CA, Biolo A, Nascimento BR, Malta DC, et al. Estatística Cardiovascular - Brasil 2020. Arq Bras Cardiol. 2020;115(3):308-439. Disponível em: https://www. scielo.br/scielo.php?script $=$ sci_arttext\&pid=S0066782X2020001100308.

26. Huntley AL, Johnson R, Purdy S, Valderas JM, Salisbury C. Measures of multimorbidity and morbidity burden for use in primary care and community settings: a systematic review and guide. Ann Fam Med. 2012;10(2):134-41.

27. Melo LA, Braga LC, Leite FPP, Bittar BF, Oséas JMF, Lima KC. Fatores associados à multimorbidade em idosos: uma revisão integrativa da literatura. Rev Bras Geriatr Gerontol. 2019;22(1):e180154.

28. Cavalcanti G, Doring M, Portella MR, Bortoluzzi EC, Mascarelo A, Dellani MP. Multimorbidity associated with polypharmacy and negative self-perception of health. Rev Bras Geriatr Gerontol. 2017;20(5):634-42.

29. World Health Organization. Mental health of older adults [Internet]. Geneva, GE: WHO; 2017 [acesso em 10 jan. 2020];[1 tela]. Disponível em: https://www. who.int/news-room/fact-sheets/detail/mental-healthof-older-adults .

30. Ramos GCF, Carneiro JA, Barbosa ATF, Mendonça JMG, Caldeira AP. Prevalência de sintomas depressivos e fatores associados em idosos no norte de Minas Gerais: um estudo de base populacional. J Bras Psiquiatr. 2015;64(2):122-31. 
31. Borges LJ, Benedetti TRB, Xavier AJ, D’orsi E. Fatores associados aos sintomas depressivos em idosos: estudo EpiFloripa. Rev Saúde Pública. 2013;47(4):701-10

32. Pinto AH, Lange C, Pastore CA, Llano PMP, Castro DP, Santos F. Capacidade funcional para atividades da vida diária de idosos da Estratégia de Saúde da Família da zona rural. Ciênc Saúde Colet. 2016;21(11):3545-55.

33. Tomás MT, Galán-Mercant A, Carnero EA, Fernandes B. Functional Capacity and Levels of Physical Activity in Aging: A 3-Year Follow-up. Front Med. 2018;4:244.
34. Campos ACV, Almeida MHM, Campos GV, Bogutchi TF. Prevalência de incapacidade funcional por gênero em idosos brasileiros: uma revisão sistemática com metanálise. Rev Bras Geriatr Gerontol. 2016;19(3):545-59.

35. Forman DE, Arena R, Boxer R, Dolansky MA, Eng JJ, Fleg JL, et al. Prioritizing Functional Capacity as a Principal End Point for Therapies Oriented to Older Adults With Cardiovascular Disease: a Scientific Statement for Healthcare Professionals From the American Heart Association. Circulation. 2017;135(16):894-918. 